\title{
Beam Loss Control on the ESS Accumulator Rings*
}

\author{
C M Warsop \\ Rutherford Appleton Laboratory, \\ Oxfordshire, UK
}

\begin{abstract}
The requirements for beam loss control on the $1.334 \mathrm{GeV}$ accumulator rings of the European Spallation Source are summarised. The main features of the beam loss collector system design are described, along with the underlying aims. Use of a specially developed code to test the proposed system under most foreseeable loss conditions, with machine errors, is described. Predicted collection efficiencies, in terms of localisation and surviving halo, are given. Simulations indicate that the required uncontrolled loss levels of $<1 \mathrm{~W} / \mathrm{m}$ over most of the machine will be achievable.
\end{abstract}

\section{INTRODUCTION}

The ESS Accelerators [1] provide $5 \mathrm{MW}$ of beam power for a short pulse length, $50 \mathrm{~Hz}$ spallation target. The $\sim 1.0 \mathrm{~ms}$ pulse from the linac is compressed to $\sim 1 \mu$ s by two accumulator rings, operating in parallel at $50 \mathrm{~Hz}$. During the $\sim 600$ turn charge-exchange injection, $2.34 \times 10^{14}$ protons are accumulated in each ring. The $1.334 \mathrm{GeV} \mathrm{H}^{-}$linac provides a mean and peak current of 70 and $114 \mathrm{~mA}$ respectively, chopped at $70 \%$ duty factor, at the revolution frequency of the rings, $1.242 \mathrm{MHz}$. This allows lossless capture in the ring RF system, maintaining a gap for extraction. After fast extraction from each ring, beams are brought together in the target transport line.

The need for low and controlled loss has dominated the machine design. Key measures for loss control in the rings include 3D collimation in the injection line, highly optimised injection with 3D painting which minimises proton foil traversals. The concern here is control of lost protons, once they enter the acceptance of the ring [2]. To allow hands on maintenance, loss levels over most of the machine (uncontrolled loss) should not exceed $1 \mathrm{~W} / \mathrm{m}$ levels. To achieve this, collimator systems localise most loss (controlled loss) in dedicated, well shielded regions of the machine.

\section{Expected and Possible Losses}

Regular losses, occurring operationally, 24 hours a day will dominate activation levels. There are unavoidable losses associated with foil interactions, expected at $0.01 \%$ levels, manifested as transverse emittance growth and energy loss. Unexpected effects, or non-optimal set up, may lead to some transverse and longitudinal loss. Important examples requiring precautions are possible space charge emittance growth and extraction loss. Also important are fault losses, where high loss levels trip off the beam. Up to a full beam pulse may require efficient removal, and fault scenarios may lead to loss in any plane. Highest priorities are for control of foil related losses, and transverse losses. For comprehensive protection, provision is also made for general longitudinal losses.

\section{LOSS COLLECTION SYSTEMS}

\section{Betatron and Momentum Tail System}

The main betatron collimation system is placed downstream from injection, in a dedicated, well shielded, dispersionless straight. For each transverse plane, the design is based on a standard two stage betatron collimation configuration [3], with long primary jaws followed by secondary jaws at relative betatron phases of $90^{\circ}$ and $163^{\circ}$. The latter is given by $180-\mu$, with $\cos \mu=\sqrt{ }\left(\varepsilon_{p} / \varepsilon_{s}\right)$. Painted emittance, primary $\left(\varepsilon_{p}\right)$, secondary $\left(\varepsilon_{s}\right)$ and aperture acceptances are $150,260,285$ and $480 \pi \mathrm{mm} \mathrm{mr}$, respectively. The secondary collimation limit is chosen on the basis of expected alignment, and also to protect the extraction system. Additional protective collimation is included at $20^{\circ}$ and $32^{\circ}$. To enhance protection, jaws are double (both sides of the beam). This configuration is efficient over a wide range of loss modes. Placement of the primary jaw $180^{\circ}$ from the injection point provides collimation of the low momentum tail generated at the foil.

\section{General Momentum System}

This consists of single stage collimation placed at the first dispersion peak after the betatron system. It is provided as a precaution against error conditions, and for removal of out-scatter from the betatron system.

\section{Practical Features and Layout}

To minimise the number of mechanical units, and enclose conveniently the most active surfaces,

\footnotetext{
* Work here based on parts of the author's Ph.D. thesis [2]
}

CP642, High Intensity and High Brightness Hadron Beams: $20^{\text {th }}$ ICFA Advanced Beam Dynamics Workshop on High Intensity and High Brightness Hadron Beams, edited by W. Chou, Y. Mori, D. Neuffer, and J.-F. Ostiguy (C) 2002 American Institute of Physics 0-7354-0097-0/02/\$19.00 
horizontal and vertical jaws are combined into a single box construction. This tubular geometry is also beneficial for interception of secondary particles and protection of downstream components. Primary jaws are of copper and secondaries of graphite, lengths are $\sim 0.5$ and $1.0 \mathrm{~m}$ respectively (three interaction lengths). The straights are enclosed in concrete; active handling concepts are included for fast removal and installation.

Compromises are made in placement of betatron collimators for combination of horizontal and vertical jaws, and to protect quadrupoles. The final betatron system consists of combined horizontal and vertical jaws at $0^{\circ}, 20^{\circ}, 32^{\circ}$ and $90^{\circ}$. The $163^{\circ}$ horizontal and vertical collimators are split around the final quadrupole to achieve near optimal phases. Phases are within about $10^{\circ}$ of ideal values. Simple collimator jaw designs are selected, flat in the longitudinal and transverse direction. The latter is strongly influenced by the rectangular machine apertures. A beam in gap kicker system could be added if required.

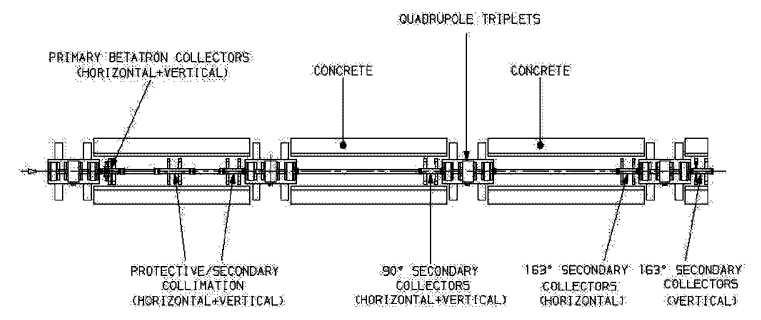

Figure 1.1 Main Betatron System Layout in Long Straight

\section{MONTE CARLO CODE}

\section{Approach and Aims}

Full understanding and control of activation on a high intensity proton machine would require complete models of (i) loss mechanisms (ii) proton loss control (iii) particle cascades and resulting distributions of unstable nuclei. The approach here is to concentrate on (ii) and model this realistically with some care. Loss mechanisms are not well known, therefore simple models of loss modes, for all expected planes and growth rates, are used. The aim here is to determine, and ensure control of, proton loss distributions: activation levels may be calculated from these.

\section{Outline of Simulation}

The code tracks protons around the machine, using a detailed lattice model with aperture geometries. Checks are made every half metre to see if a particle has been lost. Representative random magnet and alignment errors, and Q shifts are included.
Collimators are modeled as 3D objects, including interactions with inner faces, and appropriate treatment of out-scattering at boundaries. All important processes: ionisation energy loss, straggling, elastic and inelastic nuclear scattering, and multiple elastic scattering, are included. These have been checked against published data for the relevant regimes.

The aim is to ensure that the design works for most expected loss and machine error conditions. Therefore various loss conditions are defined, modelled, and loss distributions around the machine determined. The extent of surviving halos is also analysed. Basic behaviour is first assessed with no errors, and then observed over a number of runs with standard random error conditions.

\section{SIMULATION RESULTS}
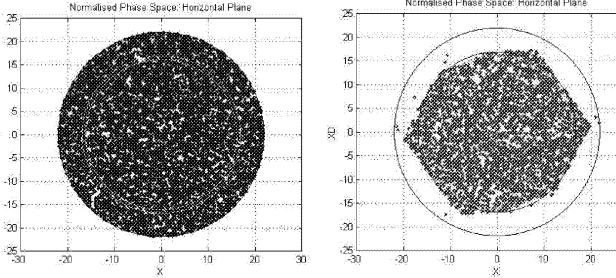

Figure 3.1 Transverse Collimation: Single Turn
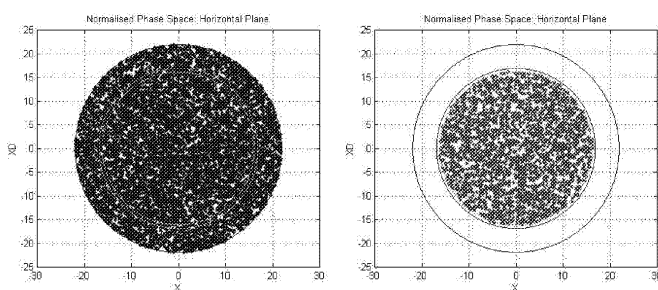

Figure 3.2 Transverse Collimation: Multiple Turn

\section{Test 1: Basic Transverse Collimation}

These tests checked the basic optical performance of the system, ensuring design compromises were reasonable. A matched beam distribution uniformly spanning the $480 \pi \mathrm{mm} \mathrm{mr}$ transverse horizontal and vertical acceptances was introduced at the primary jaw, and taken around the machine one turn. The momentum distribution was uniform over the nominal $\pm 0.8 \%$ acceptance. The test was then repeated, but over multiple turns. Simulations were also repeated a number of times with randomly generated errors.

Simulation results for single and multiple turn collimation in the horizontal plane are shown in Figures 3.1 and 3.2. These show normalised transverse phase space (axes in units $10^{-3} \mathrm{~m}^{1 / 2}$ ): input beam and 
collimated beam, with no errors. The circles indicate acceptances of 260,285 and $480 \pi \mathrm{mm} \mathrm{mr}$. The corresponding distribution of lost particles around the whole machine is shown in Figure 3.3, starting at the primary collimator. The lattice is shown schematically: darker elements are the main dipoles, indicating the main arcs of the three super period machine.

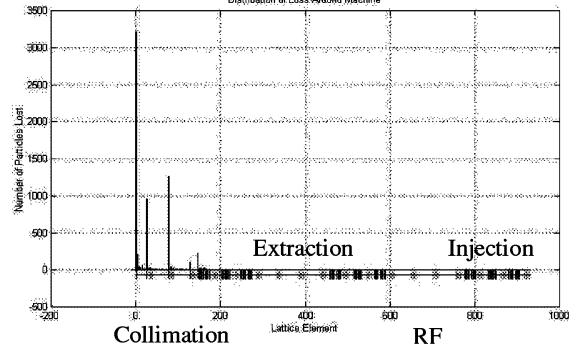

Figure 3.3 Loss Distribution for Test 1: Single Turn

These results are without machine errors: their inclusion had small effect, the most significant being an increase in surviving halo. The first figure shows the cuts made by the jaw system: in a single turn most beam is confined well within the machine acceptance, except that escaping due to out-scatter. The $99 \%$ surviving single-turn halos, without and with errors, were 344 and $374 \pm 3 \pi \mathrm{mm} \mathrm{mr}$. Corresponding numbers for multi-turn collimation were 261 and 281 $\pm 3 \pi \mathrm{mm} \mathrm{mr}$. The single turn loss distribution is given in Table 1, case 1: in the worst case with errors, $<1 \%$ escaped the collimator regions.

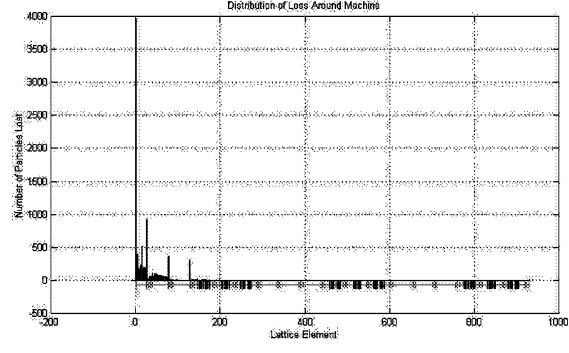

Figure 3.4 Loss Distribution for Test 2: Growth $10 \mu \mathrm{m} /$ turn

\section{Test 2: Transverse Growth Rate Tests}

These tested the operation of the betatron system for a range of transverse beam growth rates, equivalent to $1-10^{3} \mu \mathrm{m} /$ turn at the primary jaws. A beam on a single matched emittance contour was introduced at the primary collimator, transported around the machine, and the emittance then incremented on each turn. The process continued over 100 's of turns until most beam was removed. Growth rates were tested in one plane at a time: in orthogonal planes beam uniformly populated the collimated acceptance. The resulting beam loss distributions for $10 \mu \mathrm{m} /$ turn growth rates are shown in Figure 3.4, and for 10 and 1 $\mu \mathrm{m} /$ turn in cases 2 and 3 of Table 1 . These are for the horizontal plane with no errors: vertical results were essentially the same. As would be expected, spread of loss down the collimation straight increased with slower growth rates. Simulations were repeated with errors. However, worst cases with errors still achieved overall control $>95 \%$.

TABLE 1. Percentage Loss in Sections of Machine (All results without errors, except* which are 'worst cases')

\begin{tabular}{lcccc}
\hline case & $\begin{array}{c}\text { Betatron } \\
\mathbf{1}^{\text {st }} \text { Cell }\end{array}$ & $\begin{array}{c}\text { Betatron } \\
\mathbf{2}^{\text {nd }} \boldsymbol{\&} \mathbf{3}^{\text {rd }} \\
\text { Cell }\end{array}$ & $\begin{array}{c}\text { Momentum } \\
\text { Collimator } \\
\text { Region }\end{array}$ & $\begin{array}{c}\text { Rest Of } \\
\text { Machine }\end{array}$ \\
\hline 1 & $59.6 \pm 0.8$ & $37.7 \pm 0.4$ & $2.4 \pm 0.1$ & $0.3\left(0.4^{*}\right) \pm 0.1$ \\
2 & $75.0 \pm 0.8$ & $23.6 \pm 0.4$ & $1.0 \pm 0.1$ & $0.4\left(1.4^{*}\right) \pm 0.1$ \\
3 & $53.5 \pm 0.8$ & $43.3 \pm 0.4$ & $2.5 \pm 0.1$ & $0.7\left(4.6^{*}\right) \pm 0.1$ \\
\hline
\end{tabular}

\section{Test 3: Injection and Extraction Losses}

Approximate simulations of the injection process confirmed foil associated losses at $<0.01 \%$, and indicated effective loss control. Similarly, efficiency of the general momentum system was satisfactory. Simulations also showed that if unexpected halo survives until extraction, use of correction elements should allow its controlled removal.

\section{Test 4: Collector Options Studied}

Simulations, including transverse and longitudinal angles on collimator jaws, showed significant enhancements in loss control. However, improvements did not justify the increased complexity. Similarly, simulations using higher atomic mass primaries also improved localisation of loss, but not to a level that justified the increased inherent radiation hazard.

\section{CONCLUSIONS}

Studies indicate that loss control to $\geq 95 \%$ levels should be possible. Larger halos observed when errors were included showed the importance of large machine acceptances. Simulated loss distributions suggested uncontrolled loss levels peaking at $0.1 \mathrm{~W} / \mathrm{m}$ for a $1 \mathrm{~kW}$ total loss.

\section{REFERENCES}

1 The ESS Project, Volume 3, Technical Report, ISBN 389336-299-1, May 2002.

2 C M Warsop, Ph.D. Thesis, University of Sheffield, January 2002.

3 T Trenkler et al., "Principles of Two Stage Betatron and Momentum Collimation”, CERN SL/95-03 (AP). 